\title{
Aspectos micromorfológicos da interface adesiva em função da variação no preparo do espécime
}

\section{Micromorphologic aspects of the adhesive interface in function of variation in the specimen manufacturing}

Vicente de Paulo Aragão SABOIA*

Solange Katia SAITO**

Luiz André Freire PIMENTA***

\begin{abstract}
SABOIA, V. de P. A.; SAITO, S. K.; PIMENTA, L. A. F. Aspectos micromorfológicos da interface adesiva em função da variação no preparo do espécime. Pesqui Odontol Bras, v. 14, n. 4, p. 340-344, out./dez. 2000.

O objetivo deste trabalho foi mostrar os diferentes padrões micromorfológicos da interface adesiva que podem ser encontrados em fragmentos de um mesmo espécime em função da variação de fatores durante o processamento dos mesmos para análise em MEV. Para isso, cilindros de resina foram cimentados na dentina da superficie oclusal de terceiros molares inclusos recém-extraídos e posteriormente seccionados longitudinalmente para observação no MEV. Os resultados obtidos indicaram uma grande variação no direcionamento e comprimento dos "tags". Isso indica a necessidade de uma padronização dos sítios de dentina, angulação de seccionamento dos espécimes e escolha de fragmentos a serem visualizados, a fim de evitar possíveis erros de interpretação ao se analisar a eficiência da penetração dos sistemas adesivos.
\end{abstract}

UNITERMOS: Adesivos dentinários; Microscopia eletrônica de varredura.

\section{INTRODUÇÃO}

Quando a dentina intacta é exposta durante o preparo cavitário, a polpa e a cavidade oral comunicam-se pelos túbulos dentinários ${ }^{7}$. Durante os procedimentos adesivos, esses túbulos podem ser parcialmente preenchidos por monômeros hidrofilicos que se polimerizam, vedando-os e evitando a movimentação de fluidos e penetração de ácidos e produtos tóxicos para a polpa, protegendo-a, dessa forma, contra ataques externos ${ }^{1,9,13}$.

Os adesivos hidrofilicos permitem a formação de uma mistura de polimeros e componentes dentinários, criando uma interface ácido-resistente, denominada camada híbrida ${ }^{8,9,10,17}$, com a formação de "tags" de resina no interior dos túbulos dentiná$\operatorname{rios}^{4,5,6,9,10,11,12,14,16}$.

Os métodos para a visualização por microscopia eletrônica de varredura dessa interface estão bem descritos na literatura ${ }^{3,5,6,11,12,15,16}$. No entanto, podemos obter imagens diversas e até contraditórias em um mesmo espécime quando fatores como sítio de dentina para adesão, ângulo de secciona- mento do espécime e seleção do fragmento a ser visualizado são tratados de forma aleatória. Fatores como direcionamento, comprimento e densidade de "tags" são influenciados pela profundidade, tipo de dentina, bem como pela angulação no corte desta, quando do preparo do espécime ${ }^{7}$. A não-padronização dos ângulos de seccionamento da dentina e da seleção dos fragmentos de espécimes seccionados a serem visualizados podem levar a erros de interpretação na observação dos "tags" através de MEV.

O objetivo deste trabalho foi mostrar os diferentes aspectos micromorfológicos da interface adesiva que podem ser encontrados em fragmentos de um mesmo espécime a fim de orientar a padronização durante o preparo de amostras e assim minimizar erros de interpretação na análise em MEV.

\section{MATERIAIS E MÉTODOS}

Para este estudo, foram selecionados 8 terceiros molares recém-extraídos, sem cárie, que foram armazenados em formol a $10 \%$ até sua utilização. Os

\footnotetext{
*Professor da Faculdade de Odontologia da Universidade Federal do Ceará.

** Professora da Faculdade de Odontologia da Universidade de Fortaleza.

*** Professor Assistente Doutor da Área de Dentística da Faculdade de Odontologia de Piracicaba da UNICAMP.
} 
SABOIA, V. de P. A.; SAITO, S. K.; PIMENTA, L. A. F. Aspectos micromorfológicos da interface adesiva em função da variação no preparo do espécime. Pesqui Odontol Bras, v. 14, n. 4, p. 340-344, out./dez. 2000.

dentes foram desgastados por meio de lixas abrasivas de óxido de alumínio de números 320, 400 e 600 (Norton abrasivos) montadas em uma politriz giratória (Maxigrind) refrigerada com água corrente em sua porção oclusal até se obter um platô de dentina ${ }^{3}$. A raiz foi separada da coroa por meio de corte com disco diamantado montado em uma cortadeira metalográfica (Impitech PC 10), obtendo-se um disco de $4 \mathrm{~mm}$ de espessura da porção coronária. Após isso, o disco foi seccionado no sentido vestíbulo-lingual para permitir a cimentação de dois cilindros de resina composta (TPH Spectrum, lote: 31.083 , cor: B1) em sua face oclusal. Os cilindros foram obtidos por meio de inserção da resina em uma matriz bipartida de teflon de $2 \mathrm{~mm}$ de diâmetro. Para a cimentação desses cilindros, utilizou-se cimento resinoso Enforce (Dentsply, lote: 28.520) e o adesivo hidrofilico Prime $\&$ Bond 2.1 (Dentsply, lote: 32.010). A dentina foi condicionada com ácido fosfórico a 37\% durante 15 segundos, lavada por 15 segundos e então secada com papel absorvente a fim de evitar o ressecamento do substrato dentinário. Em seguida, uma camada de adesivo foi aplicada com pincel e, após 30 segundos, foi polimerizada por 20 segundos (Optilux 500 - Demetron). Uma segunda camada de adesivo foi aplicada e, após um leve jato de ar, esta foi polimerizada por 10 segundos. O cimento resinoso Enforce (cor: A3,5) foi então preparado de acordo com as instruções do fabricante. A cimentação foi realizada com pressão digital pelo mesmo operador a fim de simular um procedimento clínico de rotina. Foi realizada a polimerização final por 20 segundos em cada face.
Esse conjunto foi novamente seccionado no sentido longitudinal para permitir a obtenção de dois fragmentos, sendo um correspondente à porção voltada para a polpa (Grupo I) e o outro para a superficie externa do dente (Grupo II) (Figura 1). Para cada grupo, foram obtidos 16 espécimes, num total de 32 fragmentos. Os segmentos seccionados foram polidos usando-se uma seqüência de abrasivos com granulação decrescente de números 320, 600 e 1.000. Entre cada abrasivo, foi dado um banho em ultra-som com água destilada durante 3 minutos e um banho final de 30 minutos. A superficie plana e polida foi tratada com ácido hidroclorídrico 6 molar por 30 segundos e lavada copiosamente com água destilada. Os espécimes foram então imersos em solução de hipoclorito de sódio a $1 \%$ por 10 minutos e novamente lavados. Esse procedimento remove uma pequena quantidade de estrutura dentária entre os "tags" de resina deixando-os em alívio, permitindo assim sua melhor visualização ${ }^{11}$.

Após a metalização dos espécimes (Balzers MED 010), estes foram examinados e fotografados no microscópio eletrônico de varredura Zeiss (DSM 940A) em magnificações de 500, 1.000 e $2.000 \mathrm{X} \mathrm{a}$ $5 \mathrm{kV}$.

\section{RESULTADOS}

A avaliação da interface adesiva ao MEV revelou diferenças entre os Grupos I e II. No Grupo I (lado da polpa), foram observados "tags" longos e inclinados em direção à porção mais profunda da dentina (Figura 2). Entretanto, foram encontrados no

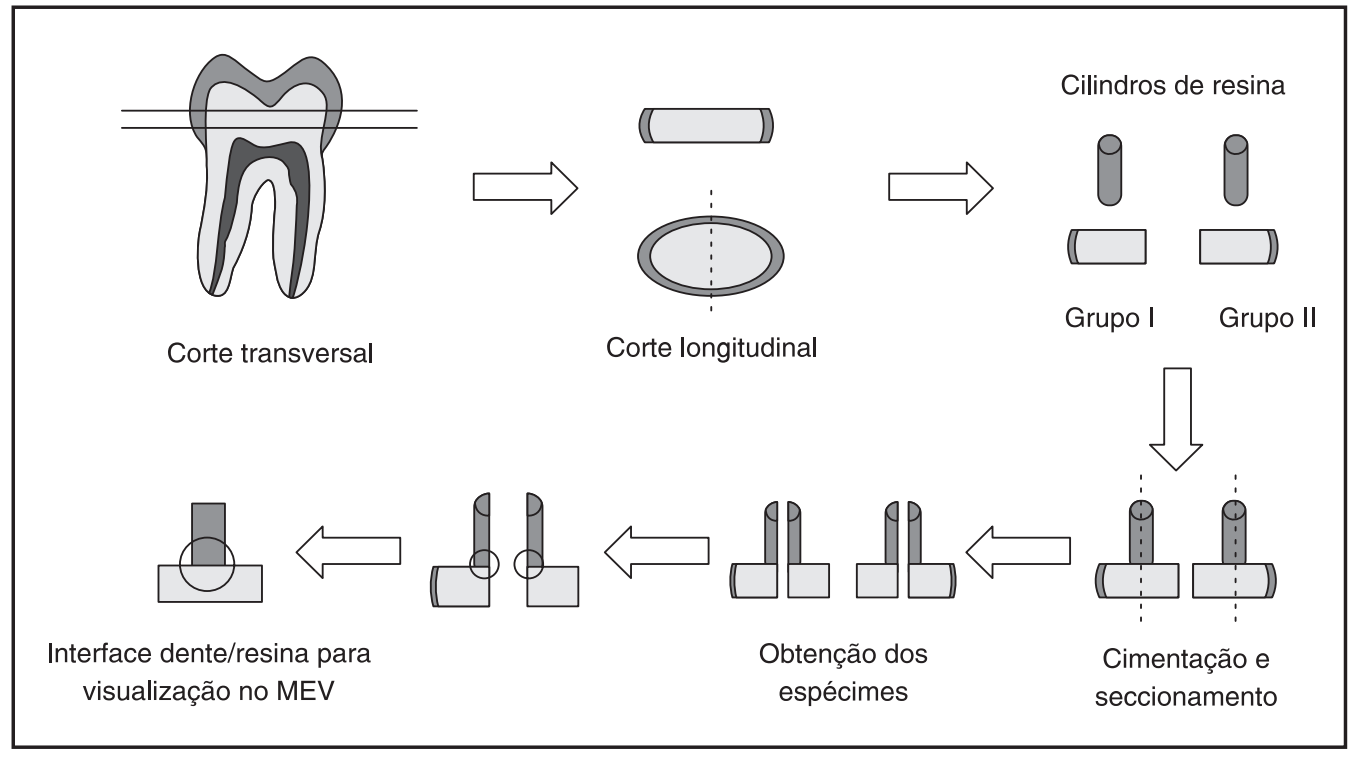

FIGURA 1 - Preparo do espécime para microscopia eletrônica. 
SABOIA, V. de P. A.; SAITO, S. K.; PIMENTA, L. A. F. Aspectos micromorfológicos da interface adesiva em função da variação no preparo do espécime. Pesqui Odontol Bras, v. 14, n. 4, p. 340-344, out./dez. 2000.

Grupo II (lado da superficie externa), na área mais superficial da dentina, "tags" mais curtos e inclinados em direção à superfície externa (Figura 3). Isso sugere que os mesmos foram cortados durante o processamento do espécime, uma vez que, na região mais profunda da dentina, visualizavam-se "tags" mais longos, porém com o mesmo direcionamento.
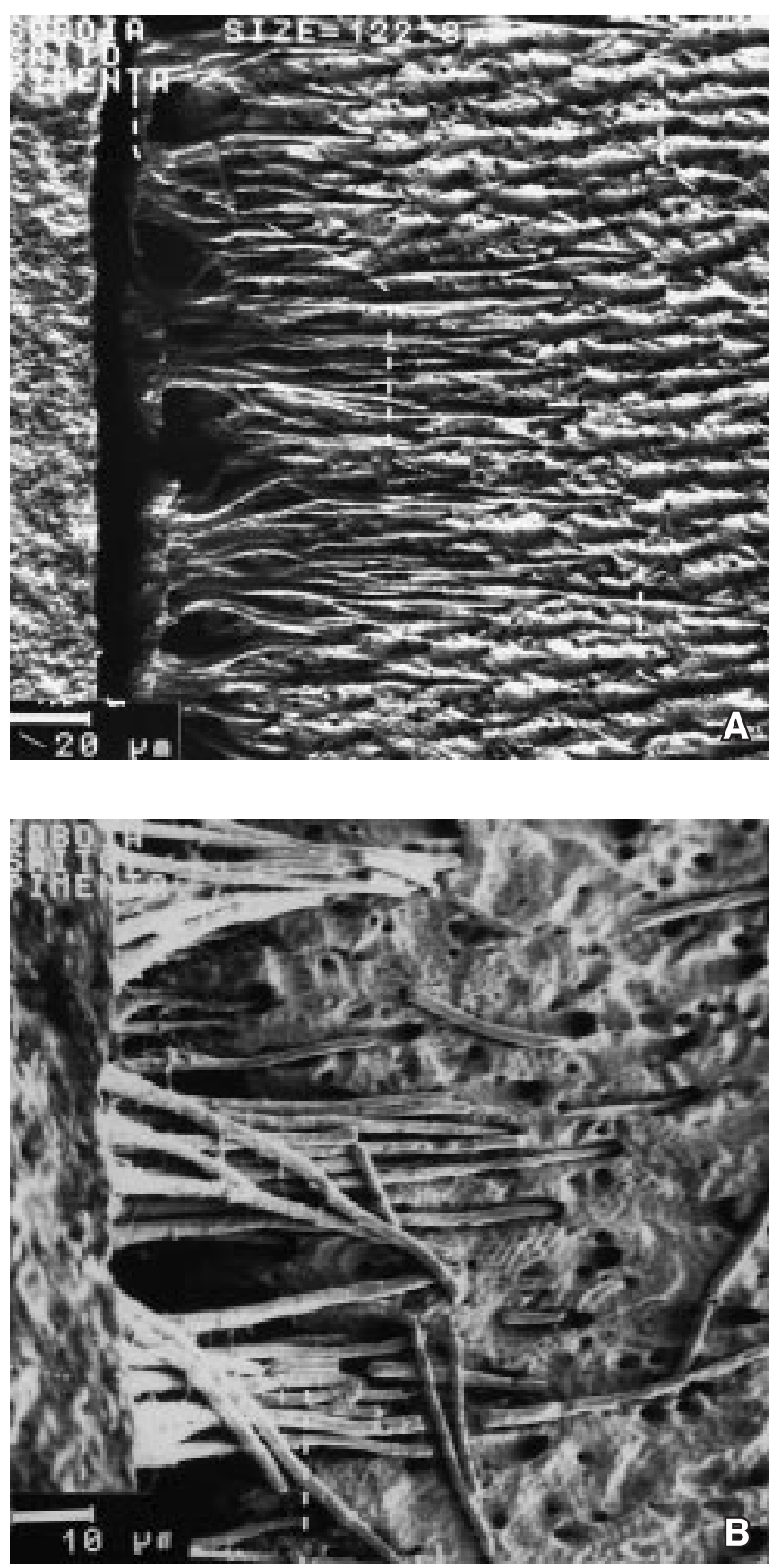

\section{DISCUSSÃO}

O direcionamento dos túbulos dentinários nas várias regiões do dente parece ter grande influência na visualização da interface adesiva ${ }^{6,7}$. No Grupo I (lado da polpa), as interfaces adesivas apresentaram "tags" integros, sugerindo que a inclinação dos túbulos em direção à polpa pode facilitar sua visualização por completo, evitando a fratura destes durante os procedimentos de corte e preparo do espécime. Isso evidencia a importância do padrão de seccionamento dos túbulos no sentido longitudinal ou o mais próximo possivel desta situação, a fim de se evitar grandes distorções na avaliação da profundidade de penetração dos "tags". Quando esse padrão de corte longitudinal é obtido, após a descalcificação da dentina, esses "tags" podem ser visualizados em sua totalidade (Figura 2).

Já para o Grupo II (lado da superficie externa), foram observados "tags" curtos na região superficial, no entanto, na porção mais profunda do corte, observou-se a presença de "tags" mais longos, porém com o mesmo padrão de inclinação. Isso pode ter ocorrido devido ao corte da dentina, seccionando os túbulos no sentido transversal ou próximo deste $^{6}$.

Pequenas variações do padrão descrito foram encontradas nos dois grupos. Isso pode ter ocorrido em função da diferença da inclinação dos túbulos entre diversos dentes ou até entre sítios de den-

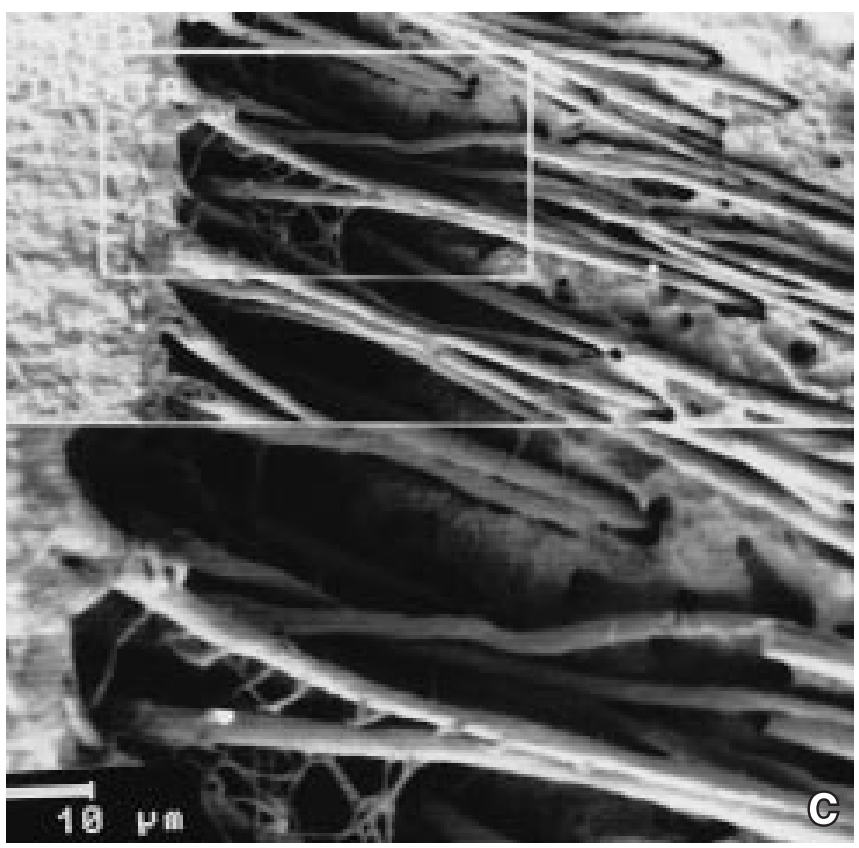

FIGURA 2 - (A: 500 X; B: 1.000 X; C: 1.000 X) Fotomicrografias dos espécimes do Grupo I (lado voltado para a polpa), mostrando longos "tags" de resina que se prolongam para o interior da dentina. 
SABOIA, V. de P. A.; SAITO, S. K.; PIMENTA, L. A. F. Aspectos micromorfológicos da interface adesiva em função da variação no preparo do espécime. Pesqui Odontol Bras, v. 14, n. 4, p. 340-344, out./dez. 2000.

tina de um mesmo dente. Muitos trabalhos na literatura ${ }^{3,5,6,11,12,15}$ descrevem a visualização da interface adesiva, mas não observam o fato de que essa região pode ser melhor visualizada em determinadas faces do dente.

PATIERNO et al. ${ }^{11}$ mostraram a interface adesiva com densos feixes de "tags" lineares medindo aproximadamente 200 micrômetros, quando fo-

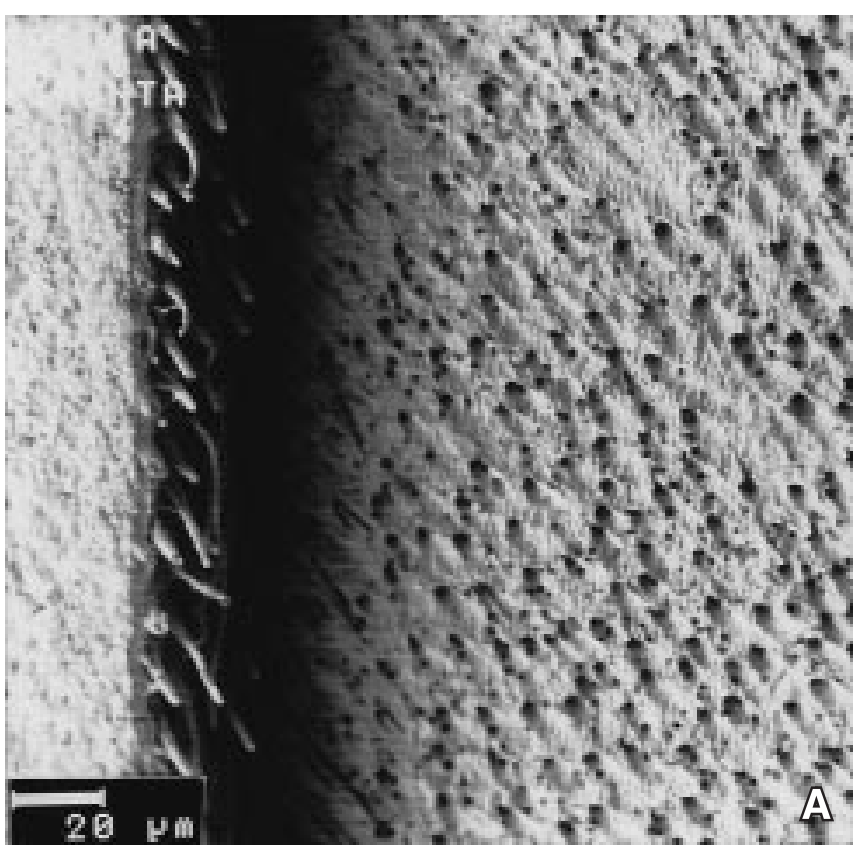

ram realizadas cimentações adesivas, não observando pequenos "tags" nessas regiões. Isso pode ser explicado pelo local usado para a adesão, no caso, a dentina cervical da parede do canal radicular, onde os túbulos estão dispostos em aproximadamente 90 graus em relação à polpa. Isso favorece uma melhor visualização dos "tags" quando realizado um corte horizontal. Nessa situação, em especial, os dois fragmentos gerados pelo seccionamento do espécime vão gerar imagens bastante semelhantes pois a disposição dos túbulos em relação ao ângulo de seccionamento favorece esta situação. A seleção criteriosa do fragmento, neste caso, não se faz tão necessária.

De acordo com esses achados, a análise da capacidade de penetração dos sistemas adesivos nos túbulos e conseqüente formação de "tags" em dentina deve ser avaliada criteriosamente. $\mathrm{O}$ aspecto micromorfológico da interface dentina-resina, especialmente da infiltração do adesivo nos túbulos dentinários ("tags") pode variar, dependendo do sítio de adesão, da angulação no seccionamento do espécime ${ }^{6}$ e da seleção do fragmento a ser observado. Estes fatores podem levar a erros de interpretação sobre a qualidade do adesivo em estudo. Após o seccionamento de um espécime, as metades obtidas podem apresentar imagens com características diferentes o que sugere a necessidade de uma padronização no processamento e seleção dos fragmentos a serem observados.
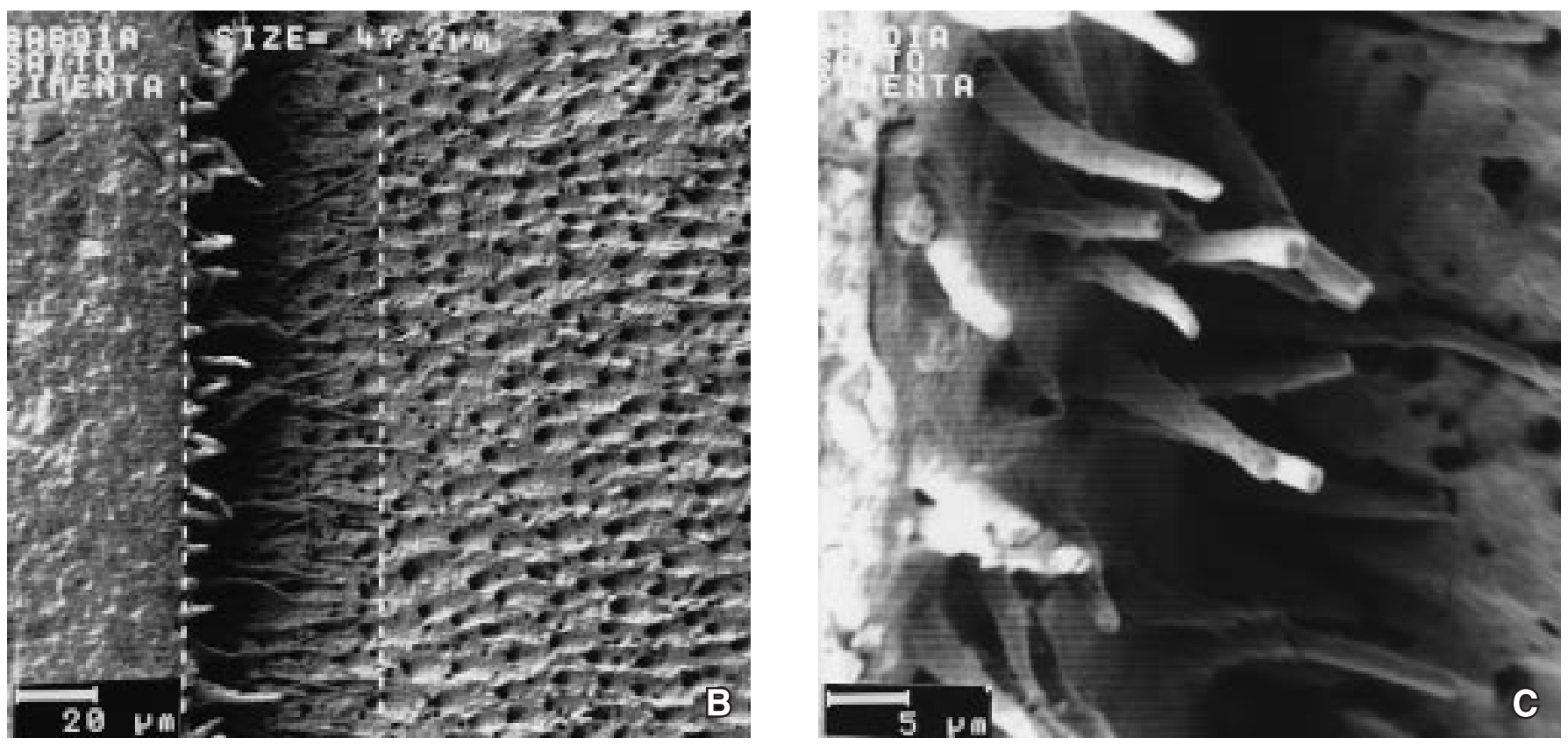

FIGURA 3 - (A: 500 X; B: 500 X; C: 2.000 X) Fotomicrografias dos espécimes do Grupo II (lado voltado para a superficie externa), mostrando "tags" superficiais curtos e fraturados e "tags" mais longos na porção mais profunda do corte. 
SABOIA, V. de P. A.; SAITO, S. K.; PIMENTA, L. A. F. Aspectos micromorfológicos da interface adesiva em função da variação no preparo do espécime. Pesqui Odontol Bras, v. 14, n. 4, p. 340-344, out./dez. 2000.

Uma melhor visualização dos "tags" pode ser obtida quando se realizam cortes longitudinais ou ligeiramente oblíquos em relação aos túbulos dentinários. Como mostrado neste trabalho, na dentina da superficie oclusal, após o seccionamento do espécime, deve-se utilizar para a visualização o lado voltado para a polpa (Grupo I). Dessa forma, pela sua inclinação em direção à polpa, os "tags" podem ser visualizados na sua totalidade, ficando protegidos durante os procedimentos de corte e preparo para análise ao MEV. A desmineralização superficial da dentina permitirá sua visualização total, evitando, assim, a fratura dos mesmos.

\section{AGRADECIMENTOS}

Ao Prof. Dr. Elliot W. Kitajima, coordenador do NAP/ESALQ-USP e Silvania Machado, pela inestimável colaboração nos procedimentos de microscopia eletrônica, sem a qual não teria sido possivel a realização deste trabalho.

SABOIA, V. de P. A.; SAITO, S. K.; PIMENTA, L. A. F. Micromorphologic aspects of the adhesive interface in function of variation in the specimen manufacturing. Pesqui Odontol Bras, v. 14, n. 4, p. 340-344, 2000.

The aim of this study was to demonstrate the different micromorphologic aspects of the adhesive interface, which can be observed in fragments of the same specimen in function of variations during the processing for scanning electron microscopy. Resin blocks were cemented on the dentin of the occlusal surfaces of recently extracted third molars and were longitudinally sectioned for observation through SEM. The results showed wide variation in the direction and length of tags. There is need for standardization of the angulation with which the specimens are cut, so as to avoid mistakes in the analysis of the penetration efficacy of adhesive systems.

UNITERMS: Dentin-bonding agents; Microscopy, electron, scanning.

\section{REFERÊNCIAS BIBLIOGRÁFICAS}

1. CAO, Z.; HUANG, L.; JIANG, J. A scanning electron microscopic observation of inner carious dentin after cleansing of the dentin-resin interface. Quintessence Int, v. 23, n. 6, p. 439-444, June 1992.

2. CHAPPEL, R. P.; EICK, J.; MORGAN, R. Shear bond strength and SEM observation of the newest dentin adhesives. J Dent Res, v. 71, p. 170 especial no. (Abstract 513), 1992.

3. CHAPPEL, R. P.; SPENCER, P.; EICK, J. D. The effects of current dentinal adhesives on the dentinal surface. Quintessence Int, v. 25, n. 12, p. 851-859, Dec. 1994.

4. EICK, J. D.; ROBINSON, S. J.; CHAPPEL, R. P. et al. The dentinal surface: its influence on dentinal adhesion. Part III. Quintessence Int, v. 24, n. 8, p. 571-582, Aug. 1993.

5. FERRARI, M. The micromorphologic relationship between resin and dentin in class V restorations: an in vivo and in vitro investigation. Quintessence Int, v. 25 , n. 9, p. 621-625, Sept. 1994.

6. FERRARI, M.; CAGIDIACO, M. C.; MASON, P. N. Micromorphologic relationship between resin and dentin in class II restorations: an in vivo and in vitro investigation by scanning electron microscopy. Quintessence Int, v. 25, n. 12, p. 861-866, Dec. 1994.

7. GARBEROGLIO, P. The ratio of the densities of dentinal tubules on the cervical and axial walls in cavities. Quintessence Int, v. 25, n. 1, p. 49-52, Jan. 1994.

8. GWINNETT, A. J. Altered tissue contribution to interface bond strength with acid conditioned dentin. Am J Dent, v. 7 , n. 5 , p. $243-246$, Oct. 1994.

9. NAKABAYASHI, N. Adhesive bonding with 4-META. Oper Dent, Supplement 5, p. 125-130, 1992.

10. NAKABAYASHI, N.; ASHIZAWA, M.; NAKAMURA, M. Identification of a resin-dentin hybrid layer in vital human dentin created in vivo: durable bonding to vital dentin. Quintessence Int, v. 23, n. 2, p. 135-141, Feb. 1992.

11. PATIERNO, J. M.; RUEGGEBERG, F. A.; ANDERSON, R. W. et al. Push-out strength and SEM evaluation of resin composite bonded to internal cervical dentin. Endod Dent Traumatol, v. 12, n. 5, p. 227-236, Oct. 1996.

12. PERDIGÃO, J.; SWIFT, E. J. Analysis of dental adhesive systems using scanning electron microscopy. Int Dent J, v. 44, n. 4, p. 349-359, Aug. 1994.

13. SANO, H.; TAKATSU, T.; CIUCCHI, B. et al. Nanoleakage: leakage within the hybrid layer. Oper Dent, v. 20, n. 1, p. 18-25, Jan./Feb. 1995.

14. SWIFT, E. J.; PERDIGÃO, J.; HEYMMAN, H. O. Bonding to enamel and dentin: a brief history and state of the art, 1995. Quintessence Int, v. 26, n. 2, p. 95-110, Feb. 1995.

15. TAY, F. R.; GWINNETT, A. J.; PANG, K. M. et al. Micromorphologic relationship of the resin-dentin interface following a total-etch technique in vivo using a dentinal bonding system. Quintessence Int, v. 26, n. 1, p. 63-70, Jan. 1995.

16. UNO, S.; FINGER, W. J. Function of the hybrid zone as a stress-absorbing layer in resin-dentin bonding. Quintessence Int, v. 26, n. 10, p. 733-738, Oct. 1995.

17. WALSHAW, P. R.; McCOMB, D. Clinical considerations for optimal dentinal bonding. Quintessence Int, v. 27, n. 9, p. 619-625, Sept. 1996.

Recebido para publicação em 28/07/99 Enviado para reformulação em 04/04/00 Aceito para publicação em 17/04/00 